\title{
Oxidative stress and peripartum outcomes (Review)
}

\author{
ZSUZSANNA SIMON-SZABO $^{1}$, ERZSEBET FOGARASI ${ }^{2}$, ENIKO NEMES-NAGY ${ }^{3}$, \\ LORAND DENES $^{4}$, MIRCEA CROITORU ${ }^{2}$ and BELA SZABO ${ }^{1}$
}

\author{
Departments of ${ }^{1}$ Obstetrics-Gynecology, ${ }^{2}$ Toxicology and Biopharmacy, ${ }^{3}$ Chemistry and Medical Biochemistry, \\ and ${ }^{4}$ Anatomy, 'George Emil Palade' University of Medicine, Pharmacy, \\ Science and Technology, 540142 Targu-Mures, Romania
}

Received February 5, 2021; Accepted March 9, 2021

DOI: $10.3892 / \mathrm{etm} .2021 .10203$

\begin{abstract}
Pregnancy, labor and childbirth are accompanied by excessive oxidative aggression. The excessive formation of free radicals [reactive oxygen species (ROS), reactive nitrogen species (RNS), chlorine reactive species (CRS)] causes cellular oxidative damage, which can be scavenged by enzymatic or non-enzymatic antioxidants in normal healthy pregnancy, physiological labor and delivery without any complications. An imbalance between the pro-oxidant and antioxidant factors may lead to oxidative stress, which contributes to the development of many diseases. This oxidative aggression can be a precursor for pathologies in the pregnant woman including eclampsia, miscarriage, preterm labor, and intrauterine growth retardation; in the offspring it may lead to bronchopulmonary dysplasia/chronic lung disease, necrotizing enterocolitis, retinopathy of prematurity, and periventricular leukomalacia. This review summarizes the studies conducted to identify the mechanisms of oxidative stress and the effect of cell membrane oxidation, the mechanisms that are behind oxidative stress-related diseases, and also those studies which have demonstrated the effect of antioxidants in preventing diseases or diminishing the effects of oxidative stress in the body, in obstetrics and neonatology.
\end{abstract}

\section{Contents}

1. Introduction

2. Oxidative stress particularities in pregnancy, during labor and birth

3. Oxidative stress-related conditions in the newborn

4. Circulating biomarkers and oxidative stress evaluation

5. Conclusions

Correspondence to: Dr Mircea Croitoru, Department of Toxicology and Biopharmacy, 'George Emil Palade' University of Medicine, Pharmacy, Science and Technology, 38 Gheorghe Marinescu Street, 540142 Targu-Mures, Romania

E-mail: mircea.croitoru@umfst.ro

Key words: oxidative stress, antioxidants, pregnancy, preterm, neonate, malondialdehyde, free radicals

\section{Introduction}

Oxidative stress is defined as an imbalance between pro-oxidant and antioxidant factors. Free radicals are molecules with one or more unpaired electrons, extremely unstable and highly reactive. When produced in excess in the body, they become mediators of cell and tissue damage, resulting in a phenomenon called oxidative stress (1).

Reactive oxygen species (ROS) (such as the superoxide anion, hydrogen peroxide, hydroxyl radical, peroxyl radical), reactive nitrogen species (RNS) [such as nitric oxide (NO), peroxynitrite, nitroxyl anion] and chlorine reactive species (CRS) (such as atomic chlorine, chlorine monoxide) have a general biological toxic effect, leading to direct peroxidation of membranes, proteins and structural enzymes, as well as nucleic acids. ROS, RNS, and CRS are molecules with free radicals or molecules without free radicals, but which can produce free radicals in certain conditions (reactive intermediates). Some free radicals, such as NO and the superoxide anion, are intentionally produced by the human body under certain conditions and at specific concentrations; they can serve as cell mediators with well-defined biological purposes (phagocytosis) (1).

Many low-molecular-weight enzymes and molecules have antioxidant capacity, protecting against these biochemical aggressors, as part of the antioxidant defense system by stabilizing free radicals (2). They are called free radical traps, but in biology and medicine are frequently referred to as antioxidants. These antioxidants can be grouped into two broad classes: endogenous (enzymatic, non-enzymatic) and exogenous (natural, synthetic) antioxidants, their mechanism of action depending on their nature (3). Most frequently, an electron transfer from the antioxidant to the free radical will stabilize the unpaired electron in the free radical, ending its highly reactive status. This transfer can take place in the form of a hydrogen atom or a covalent bond formation (4).

Total antioxidant capacity (TAC) is defined as a biological parameter, which sums up the antioxidant effects of low-molecular-weight enzymes and molecules with antioxidant properties in a living organism (5).

A panel of biomarkers of oxidative stress have been established for evaluation of the oxidative stress in pregnancy, in the fetus and the newborn (6), grouped according to their nature as: lipid peroxidation products (including 
malondialdehyde, acrolein, isoprostanes), protein oxidation products (including protein carbonyl, tyrosine related groups), DNA oxidation products, stress response proteins, a novel biomarker (microRNAs) (6), and other biomarkers, such as enzymes (myeloperoxidase), thiols (glutathione) and metallothioneins (1). Furthermore, reduced and oxidized glutathione ratio (1) and non-protein-bound iron (6) have also been used as biomarkers in general analytical determinations (6).

\section{Oxidative stress particularities in pregnancy, during labor and birth}

The study of perinatal oxidative stress is considered a priority, due to the necessity to understand the pathophysiological mechanisms behind oxidative stress-related conditions in pregnancy and neonatal life (6).

In the evaluation of oxidative stress in pregnant women and the fetus, we must consider physiological aspects, i.e., the pregnancy as a condition, which exposes the pregnant woman to increased oxidative stress, and the placenta as the source of free oxygen radicals (7).

During delivery, the neonate is transferred from an intrauterine hypoxic environment to an extrauterine normoxic one, which is believed to induce an elevated production of ROS and RNS (8).

Another aspect to be considered is the immaturity of the immune system in the fetus/newborn, concomitant with the marked exposure to the action of free radicals, the rapid increase in energy requirements after birth, the inefficient ability to achieve homeostasis, and immaturity of antioxidant capacity. These factors all predispose the newborn to significant oxidative stress (3). The inefficiency of enzymatic activity in the newborn has to be mentioned also, as it is an aspect that leads to increased oxidative stress in perinatal diseases (hypoxia, ischemia-reperfusion, activation of neutrophils and macrophages) (9).

Not only pregnancy, but labor and delivery all contribute to oxidative stress in different ways. Oxidative imbalance can pose a serious risk of complications, i.e. eclampsia, miscarriage, preterm labor, and intrauterine growth retardation (IUGR) (3,10-12).

Other authors have also reported accelerated ROS production in normal pregnancies due to increased metabolic needs (3). This increased metabolic rate allows adequate fetal growth and development, and is accompanied by enhanced oxidative stress in the placenta (13), but at the same time, it is counterbalanced by the elevated antioxidant levels (14).

Investigating oxidative stress markers at the time of birth, Arguelle et al found a positive correlation between high maternal levels of oxidative stress corresponding to even higher oxidative stress levels in newborn umbilical cord blood (15). According to Gitto et al hypoxic periods and oxidative stress periods may alternate during labor and birth, and fetal plasma has a low concentration of antioxidants to resist those oxidative damages (2). This could explain the higher oxidative stress in the neonate compared to the mother.

By investigating the type of delivery, many authors have concluded that there is the same degree of fetal oxidative stress via vaginal delivery or Caesarean section $(16,17)$. Other studies comparing vaginal delivery with Caesarean section have demonstrated that the type of delivery influences the oxidative status of the term newborn. These studies have found reduced antioxidant capacity in preterm and term infants (18-20).

According to Chiba et al increased production of oxidative stress markers is present in women with prolonged vaginal delivery and emergency Caesarean section (21).

During labor, an overwhelming oxidative imbalance can occur, which can result in ischemic reperfusion injury produced by powerful uterine contractions, affecting the neonate and predisposing them to oxidative stress (3).

Karacor et al reported significantly higher total antioxidant status (TAS) and total oxidative status (TOS) levels in pregnant women undergoing oxytocin-induced labor compared to a control group (spontaneous births). It was concluded that oxytocin used to induce childbirth is an additional stress and increases the intensity of oxidative stress and thus, antioxidant processes will be more pronounced: NO, catalase, glutathione peroxidase and glutathione were increased due to response to oxidative stress (7). NO is a biologically important molecule which can serve as an antioxidant and also as a free radical source through peroxinitrite formation; the effect depends mostly on its concentration: low levels of NO protect the organs from ischemic damage (22).

Vural et al report that oxidative stress was increased in recurrent or habitual abortion, as the antioxidant mechanisms were insufficient to compensate the increased oxidative stress; thus, oxidative stress may be involved in the etiology of abortion (23).

Some reports indicate transplacental transfer of antioxidants at the end of gestation (18).

The effectiveness of antioxidant mechanisms in neonates largely depend on the functionality of the maternal antioxidant system. The efficiency of antioxidant mechanisms can be influenced by maternal factors, i.e. age, parity, nutritional status, or environmental and genetic factors, which can cause an imbalance in the newborn in addition to their immaturity (3).

In uncomplicated pregnancies, there is a balance between the antioxidant system and reactive intermediates, but this balance can be disrupted due to complications in the pregnancy or labor with poor outcomes $(3,24)$.

Comparing oxidative stress levels in early vs. late cord clamping births, Diaz-Castro et al concluded that there is another possible oxidative stress factor in late cord clamping due to an increase in the concentration of free $\mathrm{Fe}^{2+}$ ions, especially in preterms with a lower level of iron carriers. $\mathrm{Fe}^{2+}$ is not a free radical, but it can induce the decomposition of the relatively stable hydrogen peroxide molecule, through an electron donating process, to one of the most dangerous free radicals that can be encountered in the biological environment: the hydroxyl free radical. In addition, the TAS was found to be higher in the late-clamped group, which suggests a protective effect of late cord clamping. The oxidative damage caused by ROS was found to be partly neutralized by catalase (CAT) and superoxide dismutase (SOD), sustained by the observed greater enzyme activity in the late-clamped group (25). In addition, prenatal steroid administration significantly intensified enzyme activity according to Wilinska et al and Vento et al $(26,27)$. Decreased CAT activity was reported in pregnant women undergoing instrumental delivery due to the threat of eclampsia (26). The activity of both enzymes is 
probably important because superoxide dismutase converts superoxide anion to hydrogen peroxide, a more stable molecule but with a potential to generate under certain conditions powerful oxidative damage, especially through the Fenton reaction. In a second step CAT and other enzymatic systems reduce hydrogen peroxide to water and molecular oxygen (1).

There are several studies which suggest that there are some benefits of delayed cord clamping; i.e. improvement in cardiopulmonary adaptation, lower RDS frequency, reduced incidence of intraventricular hemorrhage, lower anemia rate in infancy and longer period of breastfeeding (28-30).

A non-invasive determination method for the oxidative stress biomarkers is from saliva with the same accuracy and specificity as from plasma samples. Studying the oxidative stress biomarkers in saliva and blood samples of mothers and their newborns delivered with or without combined analgesia, Shobeiri et al did not find any statistically significant difference between the two groups (31); according to their findings, combined analgesia is not a contributing factor to oxidative stress.

In gestational diabetes, due to the metabolic disturbances during pregnancy (mitochondrial superoxide overproduction is due to elevated glucose level and endothelial cells are the main targets of the induced oxidative stress), higher levels of oxidative stress are observed. Neonates born from diabetic mothers have elevated oxidative stress parameters and decreased antioxidant capacity, compared to newborns from healthy physiological pregnancies (26).

\section{Oxidative stress-related conditions in the newborn}

There are several conditions involved in 'oxygen radical diseases in neonatology'. This category has been postulated by Saugstad and includes bronchopulmonary dysplasia/chronic lung disease, retinopathy of prematurity and necrotizing enterocolitis (32). Yet, later it was clarified that periventricular leukomalacia (33), ductus arteriosus and pulmonary circulation are affected by free radicals as well (34-36).

Demonstrating the effect of antioxidants in disease prevention or in diminishing the effects of oxidative damage, and the scientific curiosity to understand the mechanisms of action of injuries caused by free radicals, have led to widespread research in obstetrics and neonatology. Several experimental and clinical studies have warned that the use of high concentrations of $\mathrm{O}_{2}$ in neonatal resuscitation will not confer benefits in reduced hypoxic-ischemic encephalopathy and mortality, but the oxidative stress will be more elevated compared to ambient oxygen, and the guidelines recommend the use of an oxygen blender and pulse oximetry during resuscitation of the preterm, and the use of oxygen concentrations between room air and $100 \%$, adjusted according to the infant's needs to reach a saturation of $95 \%$. According to those meta-analyses and multicentric trials (37-39), neonatal mortality was reduced by $30-40 \%$ when resuscitation was carried out with $21 \%$ oxygen instead of $100 \%$ (2).

One of the possible side effects of elevated $\mathrm{O}_{2}$ exposure is the increased likelihood of leukemia, as oxygen derivates can damage DNA, and sometimes, through DNA damage, they can lead to formation of cancer cells (40).

Because neonates are at a very early stage of lung development with immaturity of the cells that are important for maturation of the airways, an inadequate antioxidant response to increased ambient oxygen and poor surfactant production have been observed (2).

ROS/RNS induce changes in the pulmonary endothelial cell structure, which leads to focal hypertrophy and altered metabolic activity. This plays a role in the pathogenesis of inflammatory pulmonary diseases, i.e. respiratory distress (RDS) of the newborn (41). Gitto et al concluded that RDS is associated with elevated ROS/RNS generation, and this leads to increased oxidative damage to the lung (2).

In premature newborns with poorly developed antioxidant defense mechanisms, even a lower concentration of supplemental oxygen may generate oxidative stress and secondary to that, lung injury. In addition, inflammatory cell accumulation and activation may generate oxidative stress, which can lead to chronic lung disease (CLD) (42). In contribution to the investigations of the mechanisms for developing CLD, Ramsay et al demonstrated that premature infants who developed CLD have oxidized specific proteins more frequently, than those without CLD (43). In their review, Gitto et al concluded that identification of these specific proteins which are more frequently oxidized in preterms who develop CLD, can lead to establishment of specific mechanisms for development of CLD (2).

As earlier studies have suggested, the main source of free radicals in the lung is phagocyte activation $(44,45)$. Bancalari and Gonzales and Speer and Groneck described in their studies that those infants who develop CLD have increased pro-inflammatory cytokine levels in their airway samples $(46,47)$. Oxidative stress can cause chronic inflammation, by activation of macrophages, which produce pro-inflammatory cytokines, such as interleukin (IL)-1 $\beta$, IL- 6 , and tumor necrosis factor (TNF)- $\alpha$. Other studies suggest that the presence of IL-10 as an anti-inflammatory cytokine in the lower airway prevents the development of CLD in preterm infants (48).

There are sufficient published data that suggest that ventilation can lead to elevated cytokine levels in neonates, and the most effective strategy is the use of nasopharyngeal continuous positive airway pressure and the avoidance of mechanical ventilation (2). Upon comparing high frequency oscillatory ventilation (HFOV) to conventional mechanical ventilation (CMV), the Cochrane Database concluded that there is no clear evidence on better outcome, when HFOV was used as elective initial ventilation strategy over CMV (2).

CLD is one of the most important factors in the mortality and morbidity of a preterm infant; especially in case of extremely low birth weight (VLBW) infants. The exposure of these immature lungs to prolonged periods of high $\mathrm{O}_{2}$ concentration is considered to be an important factor in the development of CLD. Saugstad concluded that the effect of free radicals on endothelial and epithelial cell membranes induces pulmonary edema and triggers the activation and accumulation of inflammatory cells (49).

Chitra et al found higher MDA plasma levels in neonate than in the maternal plasma. Uterine contractions produce a fluctuation in uterine blood flow (ischemia followed by reperfusion), a process known to generate free radicals. In uncomplicated births, oxidative stress is more effectively counterbalanced by the body's antioxidant power, compared to complicated births (3). 


\section{Circulating biomarkers and oxidative stress evaluation}

The evaluation of oxidative stress based on the level of peroxidation is conducted by determining malondialdehyde (MDA) levels. MDA is a product of the lipid peroxidation process along with other aldehydes and is frequently and preferentially used as a biochemical marker to assess the intensity of this process (7). In a review by Gitto et al elevated MDA concentrations were described in distressed fetuses delivered by emergency Caesarean section compared to non-distressed newborns delivered by elective Caesarean section (2). Lurie et al described that as the result of increased distress, MDA levels and glutathione oxidase activity of amniotic fluid and cord blood were elevated in case of fetuses born via emergency caesarian section, and that gender or type of anesthesia had no influence on the concentration of these biomarkers (50).

Karacor et al concluded in their study that MDA levels were significantly higher in pregnant women who underwent oxytocin-induced labor compared to the control group, which implies that the administration of oxytocin leads to maternal and fetal stress, which is why the concentration of MDA is increased (7). Another study by Schneid-Kofman et al also describes higher concentrations of MDA in pregnant women with induced labor, but with low level of the antioxidant glutathione (51). Glutathione is important in stopping the lipid peroxidation process, in which the starting free radical is regenerated and available to restart another lipid peroxidation process, by reacting with the lipid peroxide intermediates and preventing the perpetuation of the process. According to Rogers et al the increase in MDA levels in the umbilical cord blood may suggest a state of hypoxia and may also be caused by perinatal stress (52).

According to Chitra et al MDA levels in maternal and fetal plasma were significantly different. Based on this difference, birth predisposes the fetus to more intense oxidative stress compared to pregnant women, but the oxidative status of both (mother and fetus) is significantly altered (3). In a study of the oxidative status of low-birth-weight infants (LBW), Negi et al reported that MDA plasma concentrations were higher in LBW infants compared to a normal birth-weight control group, and at the same time they have found decreased levels of TAS, and vitamins $\mathrm{A}, \mathrm{E}$ and $\mathrm{C}$ (dietary antioxidants) (53).

According to Wilinska et al plasma MDA levels were elevated in full-term infants and their mothers, but MDA levels in breast milk decreased in the first days after birth, probably due to the reduced metabolic need. It was also observed that breast milk has high antioxidant content, at least in the first couple of days after birth, which is beneficial to the newborn (26).

According to Howlander et al maternal and fetal oxidative stress in cases of preeclampsia was characterized by increased serum MDA levels in both mother and fetus, compared to a healthy control group. At the same time, an increase in ROS and decrease in TOS and vitamin C levels were observed in the fetal circulation, and protein carbonyl levels were significantly higher compared to the control group (54).

TAS (also mentioned by some authors as total antioxidative capacity) and TOS (or total oxidative capacity) were studied by multiple authors. According to Alberti-Fidanza et al the diet of pregnant women significantly influenced the total antioxidant capacity, which was much higher in mothers who had a diet rich in vitamins (55). However, this should also be correlated with the meta-analysis of Bjelakovic et al who reported that high consumption of antioxidant supplements (vitamin A,E) may be associated with increased mortality, and that the potential role of vitamin $\mathrm{C}$ in decreasing life expectancy of well-nourished adults, needs further investigations. Antioxidant source should be dietary and not from supplements, the authors suggest (56).

According to Özalkaya et al TOS is much higher in cases of premature rupture of membranes and TAS is lower in cases of premature rupture of membranes and fetal inflammatory response syndrome. Also, they suggest that oxidative stress is responsible for the premature ageing of the fetal membranes of infants born before 34 weeks of gestation (57). This thesis is also supported by a number of studies carried out by Menon and Richardson (58) and Ilhan et al (59). According to Wilinska et al TAS has a declining trend in newborns during the first days of life (26).

The study of Arguelles et al concluded that based on TAS, under normal conditions, the level of maternal and fetal oxidative stress displays no significant differences, but an excess of maternal oxidative stress (caused by smoking, inappropriate lifestyle, diabetes mellitus, metabolic diseases) causes even more severe fetal oxidative stress at birth; therefore, monitoring and control of maternal oxidative stress during pregnancy is important (15). According to Howlander et al TAS was significantly lower in pregnant women with preeclampsia, which demonstrates that preeclampsia is an important perinatal pathology, which causes extra stress to both the maternal and fetal organism (54).

In the study by Seligman et al NO levels were lower in preeclamptic women than in healthy pregnant ones (60), in accordance with the findings of Conrad et al who described lower levels of NO and cGMP in preeclamptic patients (61).

In their study Karacor et al found no statistically significant difference in NO levels between the oxytocin-induced labor group and control group, thus they suggested that antioxidant mechanisms were functioning well in both groups and stress only is not enough to cause alteration in NO levels (7).

\section{Conclusions}

Upon the attempt to summarize the results of studies performed on pregnant women and newborns, we conclude that all these studies underline the need to understand the mechanisms of action of free radical formation and their action on the maternal and fetal organism, in order to identify strategies to reduce the level of oxidative stress, and minimize the harmful effects of this process on both mother and newborn.

\section{Acknowledgements}

Not applicable.

\section{Funding}

No funding was received.

\section{Availability of data and materials}

The authors of this review confirm that all information found in this review is documented by relevant references. 


\section{Authors' contributions}

ZSS performed analysis of the current published data. ZSS and MC drafted the original manuscript. EF, ENN and LD performed critical revision of the manuscript. BS provided expertise in the field of obstetrics. All authors read and approved the final manuscript.

\section{Ethics approval and consent to participate}

Not applicable.

\section{Patient consent for publication}

Not applicable.

\section{Competing interests}

The authors declare that they have no competing interests.

\section{References}

1. Fogarasi E, Croitoru MD, Fülöp I and Muntean DL: Is the oxidative stress really a disease? Acta Med Marisiensis 62 112-120, 2016.

2. Gitto E, Pellegrino S, D'Arrigo, Barberi SI and Reiter RJ: Oxidative stress in resuscitation and in ventilation of newborns. Eur Respir J 34: 1461-1469, 2009.

3. Chitra M, Mathangi DC and Johnson P: Oxidative stress during spontaneous vaginal delivery: Comparison between maternal and neonatal oxidative status. Int J Med Res 4: 60-66, 2016.

4. Reina M and Martínez A: A new free radical scavenging cascade involving melatonin and three of its metabolites (3OHM, AFMK and AMK). Comput Theor Chem 1123: 111-118, 2018.

5. Ghiselli A, Serafini M, Natella F and Scaccini C: Total antioxidant capacity as a tool to assess redox status: Critical view and experimental data. Free Radic Biol Med 29: 1106-1114, 2000.

6. Perrone S, Laschi E and Buonocore G: Biomarkers of oxidative stress in the fetus and in the newborn. Free Radic Biol Med 42: 23-31, 2019.

7. Karacor T, Sak S, Basaranoglu S, Peker N, Agacayak E, Sak ME, Turgut A, Evsen MS, Evliyaoglu O and Gul T: Assessment of oxidative stress markers in cord blood of newborns to patients with oxytocin-induced labor. J Obstet Gynaecol Res 43: 860-865, 2017.

8. Shoji $\mathrm{H}$ and Koletzko B: Oxidative stress and antioxidant protection in the perinatal period. Curr Opin Clin Nutr Metab Care 10: 324-328, 2007.

9. Perrone S, Negro S, Tataranno ML and Buonocore G: Oxidative stress and antioxidant strategies in newborns. J Matern Fetal Neonatal Med 23 (Suppl 3): S63-S65, 2010.

10. Davidge ST, Hubel CA, Brayden RD, Capeless EC and McLaughlin MK: Sera antioxidant activity in uncomplicated and preeclamptic pregnancies. Obstet Gynecol 79: 897-901, 1992.

11. Carone D, Loverro G, Greco P, Capuano F and Selvaggi L: Lipid peroxidation products and antioxidant enzymes in red blood cells during normal and diabetic pregnancy. Eur J Obstet Gynecol Reprod Biol 51: 103-109, 1993.

12. Adeoye O, Olawumi J, Opeyemi A and Christiania O: Review on the role of glutathione on oxidative stress and infertility. JBRA Assist Reprod 22: 61-66, 2018.

13. Chiarello DI, Abad C, Rojas D, Toledo F, Vázquez CM, Mate A, Sobrevia L and Marín R: Oxidative stress: Normal pregnancy versus preeclampsia. Biochim Biophys Acta Mol Basis Dis 1866: 165354, 2020.

14. Orrenius S, Gogvadze V and Zhivotovsky B: Mitochondrial oxidative stress: Implications for cell death. Annu Rev Pharmacol Toxicol 47: 143-183, 2007.

15. Arguelles S, Machado MJ, Ayala A and Machado A: Correlation between circulating biomarkers of oxidative stress of maternal and umbilical cord blood at birth. Free Radic Res 40: 565-570, 2006.
16. Fogel I, Pinchuk I, Kupferminc MJ, Lichtenberg D and Fainaru O: Oxidative stress in the fetal circulation does not depend on mode of delivery. Am J Obstet Gynecol 193: 241-246, 2005.

17. Hracsko Z, Safar ZS, Orvos H, Novak Z, Pal A and Varga IS: Evaluation of oxidative stress markers after vaginal delivery or caesarean section. In Vivo 21: 703-706, 2007.

18. Georgeson GD, Szony BJ, Steitman K, Varga IS, Kovacs A, Kovács L and László A: Antioxidant enzyme activities are decreased in preterm infants and in neonates born via caesarean section. Eur J Obstet Gynecol Reprod Biol 103: 136-139, 2002.

19. Calderon TC, Wu W, Rawson RA, Sakala EP, Sowers LC, Boskovic DS and Angeles DM: Effect of mode of birth on purine and malondialdehyde in umbilical arterial plasma in normal term newborns. J Perinatol 28: 475-481, 2008.

20. Saker M, Mokhtari NS, Merzouk SA, Merzouk H, Belarbi B and Narce M: Oxidant and antioxidant status in mothers and their newborns according to birthweight. Eur J Obstet 14: 95-99, 2008.

21. Chiba T, Omori A, Takahashi K, Tanaka K, Kudo K, Manabe M, Mariya Y and Kashiwakura I: Correlations between the detection of stress associated hormone/oxidative stress markers in umbilical cord blood and the physical condition of the mother and neonate. J Obstet Gynaecol Res 5: 958-964, 2010.

22. Gülcin I: Antioxidant activity of food constituents: An overview. Arch Toxicol 86: 345-391, 2012.

23. Vural P, Akgul C, Yildirim A and Canbaz M: Antioxidant defence in recurrent abortion. Clin Chim Acta 295: 169-177, 2000.

24. Rao G, Kamath U, Raghothama C, Pradeep KS and Rao P: Maternal and fetal indicators of oxidative stress in various obstetric complications. Indian J Clin Biochem 18: 80-86, 2003.

25. Diaz-Castro J, Florido J, Kajarabille N, Garrido-Sánchez M, Padilla C, de Paco C, Navarrete L and Ochoa JJ: The timing of cord clamping and oxidative stress in term newborns. Pediatrics 134: 257-264, 2014.

26. Wilinska M, Borszewska-Lornacka MK, Niemiec T and Jakiel G: Oxidative stress and total antioxidant status in term newborns and their mothers. Ann Agric Environ Med 22: 736-740, 2015.

27. Vento M, Aguar M, Escobar J, Arduini A, Escrig R, Brugada M, Izquierdo I, Asensi MA, Sastre S, Saenz P and Gimeno A: Antenatal steroids and antioxidant enzyme activity in preterm infants: Influence of gender and timing. Antioxid Redox Signal 11: 2945-2955, 2009.

28. McDonald SJ, Middleton P, Dowswell T and Morris PS: Effect of timing of umbilical cord clamping of term infants on maternal and neonatal outcomes. Cochrane Database Syst Rev 7: CD004074, 2013.

29. Rabe H, Diaz Rossello JL, Duley L and Dowawell T: Effect of timing of umbilical cord clamping and other strategies to influence placental transfusion at preterm birth on maternal and infant outcomes. Cochrane Database Syst Rev: Aug 15, 2012 (Epub ahead of print). doi: 10.1002/14651858.CD003248.pub3.

30. Mercer JS, Vohr BR, McGrath MM, Padbury JF, Wallach M and Oh W: Delayed cord clamping in very preterm infants reduces the incidence of intraventricular hemorrhage and late onset sepsis: A randomized controlled trial. Pediatrics 117: 1235-1242, 2006.

31. Shobeiri F, Ranjbar A, Alizadeh FG and Nazari Y: Salivary, plasma and cord blood oxidative stress biomarkers in mother and neonate: A combined analgesia concern. J Postgraduate Med Inst 31: 18-24, 2017.

32. Saugstad OD: Hypoxanthine as an indicator of hypoxia: Its role in health and disease through free radical production. Pediatr Res 23: 143-150, 1988

33. Haynes RL, Folkerth RD, Keefe RJ, Sung I, Swzeda LI, Rosenberg PA, Volpe JJ and Kinney HC: Nitrosativ and oxidative injury to premyelinating oligodendrocytes in periventricular leukomalacia. J Neuropathol Exp Neurol 62: 441-450, 2003.

34. Clyman RI, Saugstad OD and Mauray F: Reactive oxygen metabolites relax the lamb ductus arteriosus by stimulating prostaglandin production. Circ Res 64: 1-8, 1989.

35. Archer SL, Peterson D, Nelson DP, DeMaster EG, Kelly B, Eaton JW and Weir EK: Oxygen radicals and antioxidant enzymes alter pulmonary vascular reactivity in the rat lung. J Appl Physiol 66: 102-111, 1989.

36. Sanderud J, Bjoro K and Saugstad OD: Oxygen radicals stimulate thromboxane and prostacyclin synthesis and induce vasoconstiction in pig lungs. Scand J Clin Lab Invest 53: 447-455, 1993.

37. Ramji S, Rasaily R, Mishra PK, Narang A, Jayam S, Kapoor AN, Kambo I, Mathur A, Saxena NC and Saxena BN: Resuscitation of asphyxiated newborns with room air or $100 \%$ oxygen at birth: A multicentric clinical trial. Indian Pediatr 40: 510-517, 2003. 
38. Saugstad OD, Ramji S and Vento M: Resuscitation of depressed newborn infants with ambient air or pure oxygen: A metaanalysis. Neonatology 87: 27-34, 2005.

39. Tan A, Schulze AA, O'Donnell CP and Davis PG: Air versus oxygen for resuscitation of infants at birth. Cochrane Database Syst Rev 2005: CD002273, 2005.

40. Naumburg E, Belloco R, Gnattingius S and Jonzon A: Supplementary oxygen and risk of childhood lymphatic leukaemia. Acta Pediatr 91: 1328-1233, 2002.

41. Huertas JR, Palomino N, Ochoa JJ, Quiles JL, RamírezTortosa MC, Battino M, Robles R and Mataix J: Lipid peroxidation and antioxidant erythrocyte membranes of full term and preterm newborns. Biofactors 8: 133-137, 1998.

42. Welty SE: Is oxidant stress in the causal pathway of BPD? NeoReviews 1: e6-e10, 2000.

43. Ramsay PL, DeMayo FJ, Hegemier SE, Wearden ME, Smith CV and Welty SE: Clara cell secretory protein oxidation and expression in premature infants who develop bronchopulmonary dysplasia. Am J Resp Crit Care Med 164: 155-161, 2001.

44. Delacourt C, D'Ortho MP, Maquin-Mavier I, Pezet S, Housset B, Lafuma C and Harf A: Oxidant antioxidant balance in alveolar macrophages from newborn rats. Eur Respir J 9: 2517-2524, 1996

45. Pittet JF, Mackersie RC, Martin TR and Matthay MA: Biological markers of acute lung injury: Prognostic and pathogenetic significance. Am J Resp Crit Care Med 155: 1187-1205, 1997

46. Bancalari E and Gonzales A: Clinical course and lung function abnormalities during development of neonatal chronic lung disease. Bland RD and Coalson JJ (eds). In: Chronic Lung Disease in Early Infancy. Marcel Dekker, New York, NY, pp41-64, 2000.

47. Speer CP and Groneck P: Oxygen radicals, cytokines, adhesion molecules and lung injury in neonates. Semin Neonatol 3: 219-228, 1998

48. McColm JR, Stenson BJ, Biermasz N and McIntosh N: Measurement of interleukin 10 in bronchoalveolar lavage from preterm ventilated infants. ARCH Dis Child Fetal Neonatal Ed 82: F156-F159, 2000.

49. Saugstad OD: Chronic lung disease: The role of oxidative stress Biol Neonate 74 (Suppl 1): S21-S28, 1998.

50. Lurie S, Matas Z, Boaz M, Fux A, Golan A and Sadan O: Different degrees of fetal oxidative stress in elective and emergent cesarean section. Neonatology 92: 111-115, 2007.
51. Schneid-Kofman N, Silberstein T, Saphier O, Shai I, Tavor D and Burg A: Labor augmentation with oxytocin decreases glutathione level. Obstet Gynecol Int 2009: 807659, 2009.

52. Rogers MS, Mongelli JM, Tsang KH, Wang CC and Law KP: Lipid peroxidation in cord blood at birth: The effect of labor. Br J Obstet Gynaecol 105: 739-744, 1998.

53. Negi R, Pande D, Kumar A, Khanna RS and Khanna HD: Evaluation of biomarkers of oxidative stress and antioxidant capacity in the cord blood of preterm low birth weight neonates. J Matern Fetal Neonatal Med 25: 1338-1341, 2012.

54. Howlander ZH, Parveen S, Tamanna S, Khan TA and Begum F: Oxidative stress and antioxidant status in neonates born to pre-eclamptic mother. J Trop Pediatr 55: 363-367, 2009.

55. Alberti-Fidanza A, Di Renzo GC, Burini G, Antonelli G and Perriello G: Diet during pregnancy and total antioxidant capacity in maternal and umbilical cord blood. J Matern Fetal Neonatal Med 12: 59-63, 2002.

56. Bjelakovic G, Nikolova D, Gluud LL, Simonetti RG and Gluud C Mortality in randomized trials of antioxidant supplements for primary and secondary prevention: Systematic review and meta-analysis. JAMA 297: 842-857, 2007.

57. Özalkaya E, Karatekin G, Topcuoglu S, Karatepe HO, Hafizoglu T, Baran P and Ovalı F: Neonatology oxidative status in preterm infants with premature preterm rupture of membranes and fetal inflammatory response syndrome. Pediatr Neonatol 58: 437-441, 2017.

58. Menon R and Richardson LS: Preterm prelabor rupture of the membranes: A disease of the fetal membranes. Semin Perinatol 41: 409-419, 2017.

59. İlhan N, Aygun BK and Gungor H: The relationship between the latency period, infection markers, and oxidant and antioxidant states in women with preterm premature rupture of membranes. Ir J Med Sci 186: 965-970, 2017.

60. Seligman SP, Buyon JP, Clancy RM, Young BK and Abramson SB: The role of nitric oxide in the pathogenesis of preeclampsia. Am J Obstet Gynecol 171: 944-948, 1994.

61. Conrad KP, Kerchner LJ and Mosher MD: Plasma and 24-h NO(x) and cGMP during normal pregnancy and preeclampsia in women on a reduced NO(x) diet. Am J Physiol 277: F48-F57, 1999. 